\title{
Disseminação de Dados baseada em Métricas de Redes Complexas para Sistemas de Transporte Inteligentes
}

\author{
Joahannes B. D. da Costa $^{1}$, Denis Rosário ${ }^{1}$ (Co-orientador), \\ Eduardo Cerqueira ${ }^{1}$ (Orientador) \\ ${ }^{1}$ Programa de Pós-Graduação em Ciência da Computação (PPGCC) \\ Universidade Federal do Pará (UFPA) \\ Belém - Pará - Brasil \\ \{joahannes, denis, cerqueira\}@ufpa.br
}

\begin{abstract}
Some applications in Vehicular Networks (VANETs) require disseminating information among vehicles for operation. However, data dissemination in this type of network employs some challenges due to the specific characteristics of VANETs, such as high mobility of nodes and intermittent communications. Thus, this work is not only intended to create an efficient data dissemination protocol, but also to evaluate its impact on real applications. The results of simulations show that the proposed protocol offers high efficiency in terms of coverage, overhead and delay compared to widely known data dissemination protocols. In addition, when applied to a Traffic Management System (TMS), it allows vehicles to stay less time in congestion and have shorter travel time. This dissertation resulted in an article submitted to the journal and three works in conferences.
\end{abstract}

Resumo. Algumas aplicações em Redes Veiculares (VANETs) requerem informações disseminadas entre os veículos para funcionamento. Contudo, a disseminação de dados nesse tipo de rede emprega alguns desafios em função das características específicas das VANETs, como alta mobilidade dos nós e comunicações intermitentes. Assim, este trabalho não destina-se apenas na criação de um protocolo de disseminação de dados eficiente, mas também na avaliação do impacto do mesmo em aplicações reais. Os resultados de simulações mostram que o protocolo proposto oferece alta eficiência em termos de cobertura, overhead e atraso em comparação aos protocolos amplamente conhecidos de disseminação de dados. Além disso, quando aplicado a um Sistema de Gerenciamento de Trânsito (TMS), possibilita que os veículos fiquem menos tempo em congestionamentos e tenham menor tempo de viagem. Esta dissertação resultou em um artigo submetido à periódico e três trabalhos em conferências.

\section{Introdução}

As Redes Veiculares Ad hoc (do inglês, Vehicular Ad hoc Networks - VANETs) desempenham um papel importante nas aplicações de gerenciamento de trânsito (do inglês, Traffic Management System - TMS), uma vez que as informações coletadas podem ser disseminadas pelos próprios veículos até serem entregues ao TMS. No entanto, os TMSs possuem requisitos rigorosos em relação a baixa latência e à capacidade de resposta em tempo real para executar suas tarefas. Neste contexto, um protocolo eficiente de disseminação de dados é obrigatório para as operações do TMS, a fim de tomar as melhores decisões 
sobre detecção de congestionamento e reencaminhamento de veículos. No entanto, disseminar dados de forma eficiente com baixo overhead (contabilizado como o número de transmissões de mensagens) e atraso, bem como alta cobertura não é uma tarefa trivial, em função das características inerentes das VANETs, tais como alta mobilidade dos nós, densidade dinâmica e curto alcance de comunicação [Silva et al. 2016].

A forma mais simples de disseminar dados em VANETs é através da inundação da rede a partir de uma fonte para todos os veículos localizados dentro de uma Área de Interesse (AoI), e essa inundação é conhecida como Flooding. Nessa abordagem, o veículo fonte inicia a transmissão da mensagem para todos os veículos no seu alcance, e cada veículo alcançado refaz esse processo. As retransmissões ocorrem sucessivamente até que a rede seja inundada com a mensagem. O Flooding não trata do problema de tempestade de broadcast que causa sobrecarga na rede, o qual é um problema muito comum em cenários densos, devido as transmissões de mensagens redundantes ou múltiplas transmissões em um curto intervalo de tempo. Portanto, os protocolos de disseminação de dados em VANETs devem lidar com o problema de tempestade de broadcast, elevados atrasos na entrega da mensagem, baixa cobertura e colisões de pacotes na camada MAC [Cunha et al. 2016].

Realizar a disseminação de dados com base no conhecimento contextual além dos vizinhos a 1 salto provê uma seleção eficiente de nós retransmissores [Costa et al. 2017, Costa et al. 2018]. Especificamente, os dados podem ser encaminhados para o veículo identificado como um ponto comum de comunicação a partir de uma análise topológica da rede, e.g., um nó que tenha maior número de vizinhos. Nesse contexto, a rede pode ser representada através de um grafo, onde os veículos são considerados como vértices e os enlaces de comunicação entre eles como arestas. Com base nesse grafo, é possível analisar o comportamento da rede a partir de métricas de redes complexas [Zhang and Li 2015], como centralidade de grau e centralidade de intermediação. Modelar as VANETs como grafos permite avaliar as interações entre veículos e como elas impactam na rede como um todo [Alvarenga et al. 2014, Wang et al. 2017].

O restante deste artigo é organizado da seguinte forma. A Seção 2 apresenta os objetivos e principais contribuições do trabalho. A Seção 3 apresenta os trabalhos relacionados. A Seção 4 descreve o cenário de avaliação e os resultados obtidos. Na Seção 5 são descritas as publicações relacionadas ao trabalho. Por fim, a Seção 6 apresenta as conclusões.

\section{Objetivos e Principais Contribuições}

Levando em consideração os desafios e problemas levantamos, este artigo apresenta o DDRX (Disseminação de Dados baseada em métricas de Redes compleXas), um protocolo distribuído que permite disseminar dados com baixo overhead, atraso e colisões de pacotes, maximizando a cobertura. Para isso, cada veículo deve conhecer sobre seus vizinhos, a fim de identificar o melhor veículo para continuar o processo de disseminação baseado em métricas de redes complexas, tais como centralidade de grau (degree centrality) e centralidade de intermediação (betweenness centrality).

Assim, os objetivos da dissertação incluem: i) Desenvolver um protocolo que garanta alta porcentagem de entrega das mensagens, porém com um número mínimo de transmissões, baixo atraso de entrega e baixa ocorrência de colisões de pacotes na camada MAC; $i$ ) Utilizar conceitos de Teoria de Grafos e Redes Complexas para o protocolo selecionar os melhores veículos reencaminhadores das mensagens de dados; iii) Implemen- 
tar o protocolo proposto e avaliar seu desempenho através de casos de uso onde o mesmo opera tanto de forma isolada quanto em conjunto com uma aplicação de gerenciamento de trânsito.

As principais contribuições da dissertação podem ser listadas como: (i) Disseminação de dados em VANETs ciente do problema de tempestade de broadcast; e (ii) Aumento da eficiência de uma aplicação distribuída para detecção e controle de congestionamentos de veículos. Assim, podemos resumir cada uma das contribuições:

1. Disseminação de dados em VANETs ciente do problema de tempestade de broadcast.

O DDRX, descrito no Capítulo 4 de [Costa 2018], é composto de duas etapas:

(i) Criação do conhecimento de vizinhança e (ii) Seleção de retransmissores. De modo geral, o protocolo toma proveito dos beacons que são naturalmente trocados pela VANET e neles são inseridas informações adicionais, tais como o número de vizinhos que um veículo possui. Assim, com esse conhecimento local, é criado um subgrafo de diâmetro 2 que considera os vizinhos dos vizinhos do veículo que precisa disseminar uma mensagem de dados. Com o subgrafo criado, é possível identificar os melhores veículos para continuarem o processo de disseminação da mensagem a partir do uso de métricas de redes complexas, centralidade de grau e centralidade de intermediação, garantindo que um alto número de veículos recebam a mensagem com um baixo número de retransmissões da mesma. Os resultados mostram a eficiência do protocolo proposto em termos de cobertura, inserção de overhead na rede, atraso de entrega, colisão de pacotes na camada MAC em comparação com outros protocolos de disseminação de dados da literatura, conforme poderá ser visto na Seção 4 .

2. Aumento da eficiência de uma aplicação distribuída de TMS para detecção e controle de congestionamentos de veículos.

A avaliação realizada, descrita no Caso de Uso II no Capítulo 5 de [Costa 2018], utiliza uma aplicação de TMS da literatura que detecta e controla congestionamentos de veículos de forma distribuída [de Souza and Villas 2016]. O TMS é chamado de FASTER e divide suas etapas de disseminação de dados em duas, a primeira para construção do conhecimento de trânsito e a segunda responsável por indicar as decisões de reencaminhamento dos veículos. Assim, a disseminação empregada pelo FASTER, que é baseada em atraso, foi alterada para o DDRX. Foi possível observar na prática o quanto uma disseminação eficiente impacta no funcionamento das aplicações que necessitam tomar decisões de forma ágil. Os resultados obtidos mostram que o DDRX reduziu consideravelmente o overhead inserido na rede e contribuiu para tomada de decisão mais rápida por parte do TMS, conforme será visto na Seção 4

\section{Trabalhos Relacionados}

Viriyasitavat et al. [Viriyasitavat et al. 2010] propuseram o protocolo Urban Vehicular broadCAST (UV-CAST), o qual concentra-se na disseminação de dados em VANETs em cenários densos e esparsos. No UV-CAST, cada veículo pode operar em um dos dois estados: supressão de broadcast ou store-carry-forward (SCF). Quando um veículo recebe uma mensagem pela primeira vez, ele verifica se é um veículo que está na fronteira de um componente conectado. O UV-CAST assume que esses veículos têm uma maior probabilidade de conhecer novos vizinhos e, portanto, eles armazenam a mensagem e carregam-na até encontrar um novo vizinho. Por outro lado, se o veículo não é um 
veículo de fronteira, ele executa um algoritmo de supressão de broadcast para retransmitir a mensagem.

Meneguette et al. [Meneguette et al. 2014] expuseram o ALgoritmo Autônomo para Disseminação De INformações em Redes Veiculares (ALADDIN), o qual tem como principal objetivo diminuir o número de retransmissões de uma mensagem de dados, sem comprometer a cobertura. Utiliza zonas de preferência para mitigar o problema de tempestade de broadcast, que é uma área onde os veículos em seu interior são considerados mais adequados para disseminar a mensagem, assim como para alcançar o maior número de vizinhos. E também, o conceito de Computação Autonômica, que é utilizada para decisão de quando retransmitir uma mensagem de dados, baseado em uma eficiência de propagação calculada a partir do número de mensagens transmitidas e o número de beacons recebidos em cada veículo, fazendo com que o veículo saiba quando retransmitir ou manter a mensagem.

Akabane et al. [Akabane et al. 2016] apresentaram o protocolo de disseminação de dados em cenários urbanos e rodoviários denominado Context-Aware Routing $p R O$ tocol (CARRO). Tal protocolo explora o conhecimento do contexto geográfico para a disseminação em VANETs. O CARRO seleciona veículos localizados em setores geográficos de alta prioridade em seu raio de comunicação para continuar o processo de disseminação. Considera também, o mecanismo de store-carry-forward assim que o número de veículos na mesma área geográfica não é satisfatório para continuar o processo de disseminação. Cada veículo troca beacons periodicamente para obter informações de contexto sobre os veículos vizinhos a 1 salto, onde nos beacons são enviadas informações de direção, cenário em que o veículo se encontra, seu id e se o mesmo possui a mensagem.

Cunha et al. [Cunha et al. 2014] introduziram o protocolo Clustering Coefficient and node DEGREE (CC-DEGREE), o qual identifica os melhores nós retransmissores com base em duas métricas: i) o coeficiente de agrupamento, o qual representa o número de conexões entre um veículo vizinho dividido pelo número total de conexões possíveis entre os vizinhos do veículo; ii) grau do nó, que representa o número de vizinhos a 1 salto que este nó possui. A partir dessas duas métricas, tempos para agendamento de retransmissão são atribuídos para veículos continuarem o processo de disseminação.

Com base na análise de protocolos de disseminação de dados, foi possível concluir que é essencial ter alta cobertura para disseminação de dados, mas com baixo overhead e atraso no processo de retransmissão. Isso envolve estar ciente do conhecimento contextual além dos vizinhos de 1 salto sem aumentar o overhead para aprimorar a decisão de seleção do retransmissor, permitindo obter uma cobertura alta com baixo overhead e atraso. A Tabela 1 resume os protocolos de disseminação de dados para VANETs analisados, com adição do protocolo proposto DDRX. Tais protocolos foram classificados com base em dois conjuntos de critérios: estratégia de encaminhamento utilizada e pressupostos necessários para a operação do protocolo.

\section{Avaliação e Resultados Obtidos}

As simulações para avaliar o desempenho do DDRX foram realizadas no framework VEINS ${ }^{1}$ do OMNeT++2 o qual implementa a pilha de protocolo do padrão IEEE 802.11p. Para estabelecer um cenário de avaliação, utilizou-se dez ruas de duas pistas em uma área

\footnotetext{
1http://veins.car2x.org/

2 http://omnetpp.org/
} 
Tabela 1. Resumo dos protocolos de disseminação de dados

\begin{tabular}{|c|c|c|c|c|c|c|c|}
\hline \multirow[b]{2}{*}{ Protocolo } & \multicolumn{4}{|c|}{ Estratégia de encaminhamento utilizada } & \multicolumn{3}{|c|}{ Suposições requeridas } \\
\hline & Posição & SCF & Distância & Topologia local & Beacon & GPS & $\begin{array}{l}\text { Posição dos } \\
\text { vizinhos }\end{array}$ \\
\hline UV-CAST & & $\checkmark$ & $\checkmark$ & & & $\checkmark$ & \\
\hline ALLADIN & $\checkmark$ & $\checkmark$ & $\checkmark$ & & $\checkmark$ & $\checkmark$ & \\
\hline CARRO & $\checkmark$ & $\checkmark$ & $\checkmark$ & & $\checkmark$ & $\checkmark$ & \\
\hline CC-DEGREE & $\checkmark$ & $\checkmark$ & $\checkmark$ & $\checkmark$ & $\checkmark$ & $\checkmark$ & $\checkmark$ \\
\hline DDRX & $\checkmark$ & & $\checkmark$ & $\checkmark$ & $\checkmark$ & $\checkmark$ & $\checkmark$ \\
\hline
\end{tabular}

de $1 \mathrm{Km}^{2}$ da cidade de Manhattan nos Estados Unidos, obtido através do OpenStreetMap $\mathrm{s}^{3}$ e importada pelo Simulation of Urban MObility (SUMO) ${ }^{4}$ para gerar os registros de movimentação dos veículos. Para quantificar a evolução do tráfego neste cenário, variou-se a densidade de veículos em 100,200,300,400,500,600 e 700 veículos $/ \mathrm{km}^{2}$, na avaliação em que o DDRX opera de forma isolada. Por outro lado, para avaliar o DDRX em funcionamento no TMS, variou-se a densidade veicular em 500, 700, 900 e 1100 veículos $/ \mathrm{km}^{2}$ para forçar a ocorrência de congestionamentos no cenário.

A Figura 1 apresenta os principais resultados para a disseminação de dados em VANETs, que é a cobertura e o número de retransmissões da mensagem (overhead) pelos protocolos DDRX, CARRO, UV-CAST, CC-DEGREE e Flooding. Pode-se observar nos resultados da Figura 1(a) que todos os protocolos atingem pelo menos $94.8 \%$ de cobertura em densidade mais baixa e acima de $98 \%$ em densidade mais alta, taxa de cobertura aceitável para protocolos de disseminação em VANETs. A menor cobertura do DDRX em comparação aos demais protocolos nas densidades mais baixas é justificado pelo fato de que os protocolos UV-CAST, CC-DEGREE e CARRO consideram o mecanismo de store-carry-forward para garantia de entrega das mensagens, o qual adicionam um atraso na entrega das mensagens. As altas taxas de cobertura alcançadas pelo Flooding são reflexo da falta de seleção de nós retransmissores, impactando no aumento do número de transmissões tal como apresentado na Figura 1(b). A Figura 1(b) resume a eficiência do DDRX, onde o mesmo apresenta a menor taxa de retransmissões da mensagem mantendo uma alta cobertura. O DDRX diminui o número de retransmissões em $90.25 \%$, $64.10 \%, 57.12 \%$ e $48.92 \%$ em relação aos protocolos Flooding, CARRO, UV-CAST e CC-DEGREE, respectivamente. Isso é resultado da eficiente seleção de nós retransmissores e com isso o DDRX conseguiu cumprir seu objetivo inicial. Ainda, o DDRX consegue disseminar as mensagens com atraso $51.22 \%$ menor e causar $41.71 \%$ menos colisões de pacotes na camada MAC (mitigação do problema de tempestade de broadcast) em comparação aos demais protocolos avaliados, conforme pode ser visto com mais detalhes no Capítulo 5 de [Costa 2018].

A Figura 2 exibe os principais resultados para avaliar a eficiência da aplicação do DDRX no TMS denominado de FASTER, o que foi chamado de FASTER-DDRX. Pode-se observar na Figura 2(a) que o FASTER-DDRX transmite cerca de 95\% menos mensagens que o FASTER no processo de envio da decisão de reencaminhamento dos veículos, ou seja, no reroteamento dos mesmos. Portanto, o FASTER-DDRX consegue disseminar em todo o cenário as informações de tráfego das vias inserindo um menor overhead na rede e sem comprometer o bom funcionamento do FASTER. Por outro lado,

$\sqrt[3]{\text { http: / / www.openstreetmap.org/ }}$

http://sumo.dlr.de 


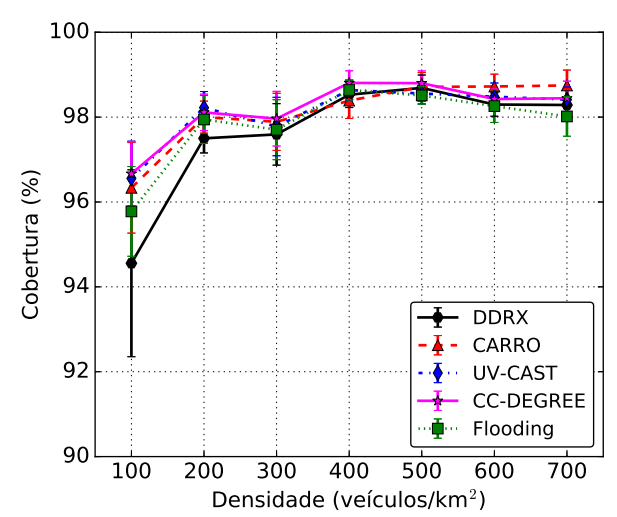

(a) Cobertura

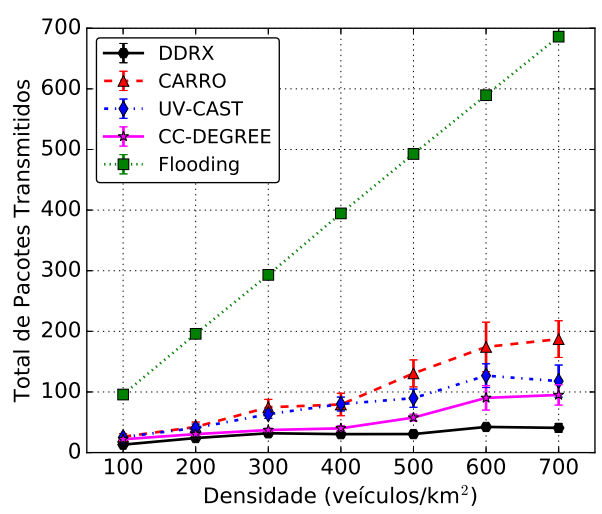

(b) Número de Mensagens Transmitidas

Figura 1. Resultados de Simulação para Disseminação de Dados

a Figura 2(b) exibe o tempo médio que os veículos ficam parados em congestionamentos, permitindo avaliar a eficiência do algoritmo de roteamento de veículos em relação às condições de tráfego veicular identificadas. Pode-se perceber que com o FASTER-DDRX os veículos ficam menos tempo em congestionamentos. Nas densidades de 500, 700 e 900 veículos $/ \mathrm{km}^{2}$ o FASTER-DDRX tem resultados próximos ao FASTER, e ambos possuem menor tempo de congestionamento quando comparados à Mobilidade Original do Cenário (MOC), que é a mobilidade do cenário sem nenhuma estratégia de reencaminhamento de veículos. Já na densidade de 900 e 1100 veículos $/ \mathrm{km}^{2}$, o FASTER-DDRX tem resultados superiores a MOC e FASTER. Isso se justifica pela disseminação eficiente empregada pelo DDRX, onde as informações acerca das condições de trânsito são disseminadas com menor atraso e alta cobertura, especialmente quando a rede está mais densa e a seleção de nós retransmissores possui mais opções de candidatos. Essa análise apresenta que FASTER-DDRX possui desempenho semelhante e até superior ao FASTER, em alguns casos, reiterando a eficiência de disseminação do protocolo DDRX.

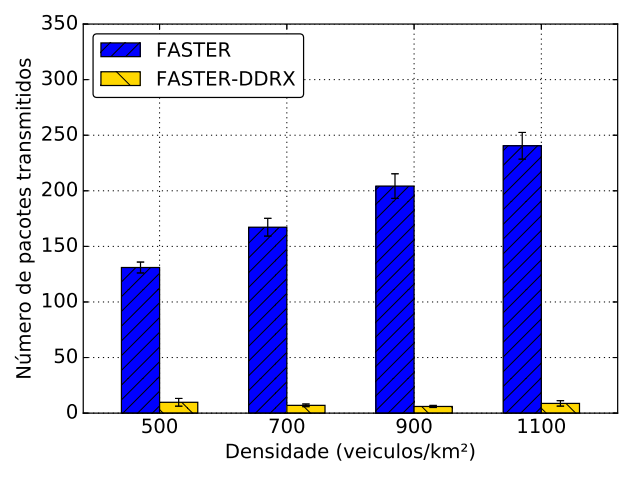

(a) Número de Transmissões

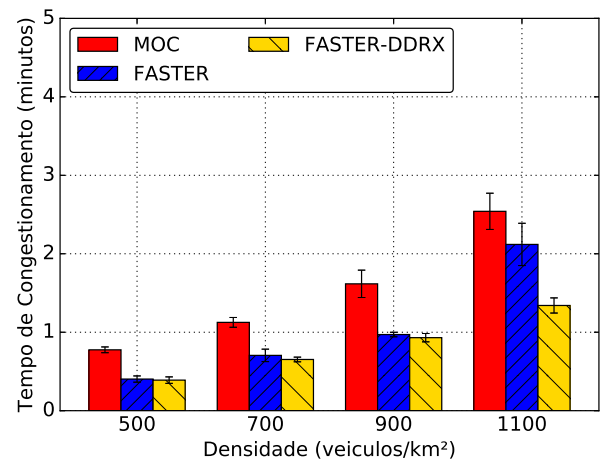

(b) Tempo em Congestionamento

\section{Figura 2. Resultados de Simulação para Disseminação de Dados na aplicação de TMS}

Com base nos resultados obtidos, pode-se concluir que o DDRX é um protocolo eficiente para disseminação de dados tanto operando de forma isolada quanto como parte de uma aplicação de TMS que exige uma disseminação de dados eficiente para seu funcionamento. Ademais, avaliações adicionais de gerenciamento de trânsito como Cobertura, 
Número de Transmissões para Construção do Conhecimento de Trânsito, Tempo Total de Viagem e Velocidade Média podem ser consultados na Subseção 5.2.2 do Capítulo 5 de [Costa 2018].

\section{Publicações}

Como resultado desta dissertação de mestrado, foram publicados dois artigos em conferências internacionais de prestígio e grande impacto na área de redes e sistemas distribuídos. Um artigo foi publicado no Simpósio Brasileiro de Redes de Computadores e Sistemas Distribuídos (SBRC 2018), o qual é o principal evento nacional da área. Por fim, um artigo foi submetido para um periódico internacional, como convite dos melhores artigos aceitos no SBRC 2018. Vale a pena realçar que todos os artigos publicados em conferências internacionais estão classificados no extrato superior da CAPES-CC (de B1 a A1). Assim, segue a lista em detalhes dos artigos aceitos e submetidos.

\section{Periódicos:}

- 2018 - Springer Journal of Internet Services and Applications, Qualis B1, CiteScore 3.25. (Submetido)

\section{Conferências:}

- 2017 - IEEE International Symposium on Network Computing and Applications, Qualis B1.

- 2018 - Simpósio Brasileiro de Redes de Computadores e Sistemas Distribuídos, Qualis B2.

- 2018 - IEEE International Symposium on Computers and Communications, Qualis A2.

\section{Conclusão}

Este artigo resume as contribuições da dissertação de mestrado em [Costa 2018]. O estado da arte em disseminação de dados não consideram a análise topológica local para criação de índices que ajudam na seleção dos melhores veículos para continuarem o processo de disseminação de dados. Ainda, as abordagens não empregam soluções para tratar os problemas de comunicação das VANETs, o que muita das vezes podem sobrecarregar a rede com transmissões desnecessárias e introduzir atraso indesejado nessa entrega dos dados.

Em resumo, aplicações em VANETs necessitam que os dados sejam disseminados na rede para execução de suas tarefas. Porém, esta é uma tarefa desafiadora devido as características específicas das VANETs, tais como, mobilidade altamente dinâmica, tempo curto de contato entre os veículos e comunicação de curto alcance. Desta forma, foi apresentado o protocolo DDRX para disseminação de dados com baixo overhead, colisões e atraso, mantendo a alta cobertura. No DDRX, cada veículo deve manter o conhecimento local de seus vizinhos de 1 e 2 saltos, que serão usados para construção de um grafo de diâmetro 2. Com o grafo criado, métricas de redes complexas são utilizadas a fim de identificar os melhores veículos para retransmitirem a mensagem. A solução proposta avança o estado da arte na utilização de métricas de redes complexas para identificação de veículos retransmissores.

\section{Referências}

[Akabane et al. 2016] Akabane, A., Pazzi, R., Madeira, E., and Villas, L. A. (2016). CARRO: A Context-Awareness Protocol for Data Dissemination in Urban and Highway Scenarios. In Proceedings of the 8th IEEE Latin-American Conference on Communications (LATINCOM'16), pages 1-6. IEEE. 
[Alvarenga et al. 2014] Alvarenga, D., da Cunha, F. D., Viana, A. C., Mini, R. A., and Loureiro, A. A. (2014). Classificando comportamentos sociais em redes veiculares. In Anais do XXXIV Simpósio Brasileiro de Redes de Computadores e Sistemas Distribuídos (SBRC'14).

[Costa 2018] Costa, J. (2018). Disseminação de dados baseada em métricas de redes complexas para sistemas de transporte inteligentes. Master's thesis, Universidade Federal do Pará.

[Costa et al. 2017] Costa, J., Lobato, W., de Souza, A. M., Rosário, D., Villas, L. A., and Cerqueira, E. (2017). Centrality-based data dissemination protocol for vehicular ad hoc networks. In 2017 IEEE 16th International Symposium on Network Computing and Applications (NCA), pages 1-4. IEEE.

[Costa et al. 2018] Costa, J., Rosário, D., de Souza, A. M., Villas, L. A., and Cerqueira, E. (2018). Data dissemination based on complex networks' metrics for distributed traffic management systems. In 2018 IEEE Symposium on Computers and Communications (ISCC), pages 01062-01067. IEEE.

[Cunha et al. 2014] Cunha, F., Maia, G., Viana, A., Mini, R., Villas, L. A., and Loureiro, A. (2014). Socially inspired data dissemination for vehicular ad hoc networks. In Proceedings of the 17th ACM international conference on Modeling, analysis and simulation of wireless and mobile systems, pages 81-85. ACM.

[Cunha et al. 2016] Cunha, F., Villas, L. A., Boukerche, A., Maia, G., Viana, A., Mini, R., and Loureiro, A. (2016). Data communication in vanets: Protocols, applications and challenges. Ad Hoc Networks, 44:90-103.

[de Souza and Villas 2016] de Souza, A. M. and Villas, L. A. (2016). A fully-distributed traffic management system to improve the overall traffic efficiency. In Proceedings of the 19th ACM International Conference on Modeling, Analysis and Simulation of Wireless and Mobile Systems, pages 19-26. ACM.

[Meneguette et al. 2014] Meneguette, R. I., Maia, G., Madeira, E. R., Pinto, A. R., Loureiro, A. A., and Villas, L. A. (2014). Um algoritmo autônomo para disseminação de informações em redes veiculares. In Anais do Simpósio Brasileiro de Redes de Computadores e Sistemas Distribuídos (SBRC'14).

[Silva et al. 2016] Silva, F., Boukerche, A., Silva, T., Ruiz, L., Cerqueira, E., and Loureiro, A. (2016). Vehicular networks: a new challenge for content-delivery-based applications. ACM Computing Surveys (CSUR), 49(1):11.

[Viriyasitavat et al. 2010] Viriyasitavat, W., Bai, F., and Tonguz, O. (2010). UV-CAST: an Urban Vehicular Broadcast Protocol. In Proceedings of the IEEE Vehicular Networking Conference (VNC'10), pages 25-32. IEEE.

[Wang et al. 2017] Wang, J., Jiang, C., Zhang, K., Quek, T. Q., Ren, Y., and Hanzo, L. (2017). Vehicular sensing networks in a smart city: Principles, technologies and applications. IEEE Wireless Communications.

[Zhang and Li 2015] Zhang, H. and Li, J. (2015). Topology analysis of vanet based on complex network. In LISS 2014, pages 1143-1148. Springer. 